\title{
Finite groups in which normality, permutability or Sylow permutability is transitive
}

\author{
Izabela Agata Malinowska
}

\begin{abstract}
Y. Li gave a characterization of the class of finite soluble groups in which every subnormal subgroup is normal by means of $N E$-subgroups: a subgroup $H$ of a group $G$ is called an $N E$-subgroup of $G$ if $N_{G}(H) \cap$ $H^{G}=H$. We obtain a new characterization of these groups related to the local Wielandt subgroup. We also give characterizations of the classes of finite soluble groups in which every subnormal subgroup is permutable or Sylow permutable in terms of $N E$-subgroups.
\end{abstract}

\section{Introduction and notation}

All groups considered here are finite. Characterizations and criteria for a group to belong to a particular class of groups are still studied by many mathematicians. Many of these characterizations or criteria depend specifically on some subgroup embedding properties. The first nontrivial result in this area seems to be the theorem of Dedekind on the structure of groups with all subgroups normal. Such groups are now called Dedekind groups. Investigation of classes of groups by using subgroup embedding properties is still applied and is the central theme of monographs, see for example [5]. In this article we determine how the embedding properties of several families of subgroups of a group influence the structure of the group.

Key Words: $N E$-subgroup, $T$-group, $P T$-group, $P S T$-group, local Wielandt subgroup. 2010 Mathematics Subject Classification: Primary 20D10,20D20; Secondary 20D25. Received: 10 April, 2013

Revised: 1 October, 2013

Accepted: 14 October, 2013 
We use conventional notions and notations, as in $[5,18]$. Throughout this paper $G$ stands for a finite group and $\pi(G)$ denotes the set of primes dividing $|G|$. For a prime $p, \operatorname{Syl}_{p}(G)$ is the set of Sylow $p$-subgroups of $G$. A subgroup $H$ of $G$ is said to be permutable in $G$ if $H$ permutes with every subgroup of $G$. A group $G$ is said to be a $P T$-group (respectively, $T$-group) if permutability (respectively, normality) is a transitive relation in $G$. The first explicit mentioning of $T$-groups in the literature was in the forties of 20 th century. They are a natural generalization of Dedekind groups, since $T$-groups are exactly those groups where all subnormal subgroups are normal. By a result of Ore ([5]) $P T$-groups are exactly those groups where all subnormal subgroups are permutable. A subgroup of $G$ is called s-permutable in $G$ if it permutes with all Sylow subgroups of $G$. A group $G$ is said to be a PSTgroup if $s$-permutability is a transitive relation in $G$. By a result of Kegel [5, Theorem 1.2.14(3)], PST-groups are exactly those groups where all subnormal subgroups are $s$-permutable. There are several characterizations in the literature of finite soluble $T$-groups, $P T$-groups and $P S T$-groups (see for example $[5,15])$.

The basic structure of soluble $T$-groups, $P T$-groups and $P S T$-groups was established by Gaschütz, Zacher and Agrawal; this result shows that the classes of all soluble $T$-, $P T$-, and $P S T$-groups are closed under taking subgroups and under taking epimorphic images (see [5]). Since a simple nonabelian group is a $T$-group the first assertion is not true for the class of all $T$-groups $(P T$ - or $P S T$-groups).

Recall that, for a prime $p$, a group $G$ satisfies the property $\mathcal{C}_{p}$ if every subgroup of a Sylow $p$-subgroup $P$ of $G$ is normal in its normalizer $N_{G}(P)$, that $G$ satisfies $\mathcal{X}_{p}$ if every subgroup of a Sylow $p$-subgroup $P$ of $G$ is permutable in $N_{G}(P)$, and that $G$ satisfies $\mathcal{Y}_{p}$ if whenever $H$ and $K$ are $p$-subgroups of $G$ with $H \leqslant K, H$ is $s$-permutable in $N_{G}(K)$. The properties $\mathcal{C}_{p}, \mathcal{X}_{p}, \mathcal{Y}_{p}$ are inherited by subgroups (see [5, page 68]) and they are closely related to soluble $T$-groups, $P T$-groups and $P S T$-groups (see [5]).

Theorem 1.1. Let $G$ be a group. Then:

(1) $G$ is a soluble PT-group if and only if $G$ satisfies $\mathcal{X}_{p}$ for all $p \in \pi(G)$ ([7], Theorem A);

(2) $G$ is a soluble PST-group if and only if $G$ satisfies $\mathcal{Y}_{p}$ for all $p \in \pi(G)$ ([4], Theorem 4);

(3) $G$ is a soluble PT-group if and only if $G$ is a soluble PST-group whose Sylow subgroups are Iwasawa ([5], Corollary 2.1.12 (1)).

In [9] Berkovich investigated the following concept: a subgroup $H$ of a finite group $G$ is called an NR-subgroup (Normal Restriction) if, whenever 
$K \unlhd H, K^{G} \cap H=K$, where $K^{G}$ is the normal closure of $K$ in $G$. He also proved that, if all Sylow subgroups of a group $G$ are $N R$-subgroups, then $G$ is supersoluble. The notion of $N R$-subgroup is equivalent to the notion of normal sensitive subgroup (see [6]) which is in fact equivalent to the notion of $C E P$-subgroup (congruence extension property), which is even older and given in other algebraic structures (see [11]). A subgroup $H$ of a group $G$ is called an $N E$-subgroup of $G$ if $N_{G}(H) \cap H^{G}=H$ (see [14]). $N R$-subgroups and $N E$-subgroups were used for characterizations of soluble $T$-groups (see $[6,15,16])$.

One of the purposes of this paper is to characterize soluble PST-groups and $P T$-groups in terms of $N E$-subgroups. These results are inspired by the results [5, Corollary 2.2.24] and [7, Theorem D] (see Lemma 2.2). We show that some subgroup embedding properties, which have been used to characterize soluble T-groups, can also be used to characterize soluble PT- and PSTgroups. In [17] we gave the characterization of soluble PST-groups in terms of $N R$-subgroups. In this paper we give another proof of it. We also characterize soluble $P T$-groups in terms of $N R$-subgroups.

The second purpose of this paper is to obtain new characterizations of $\mathcal{C}_{p}$-groups and soluble $T$-groups related to the local Wielandt subgroup. The motivation for these results comes from results of Bryce, Cossey, BallesterBolinches, Esteban-Romero and Kaplan [10, 4, 13]. We obtain a characterization of $\mathcal{C}_{p}$-groups (Theorem 3.6) like Kaplan's characterization of soluble $T$-groups [13]. We also observe that Kaplan's result [13, Theorem 1] related to the Wielandt subgroups of metabelian groups extends to the class of all soluble groups of $p$-length one for all $p$.

\section{Groups in which permutability or Sylow permutability is transitive}

We say that a group $G$ satisfies $\overline{N E}_{p}$ if every normal subgroup of a Sylow $p$-subgroup of $G$ is an $N E$-subgroup of $G$. A group $G$ satisfies $N R_{p}$ if a Sylow $p$-subgroup of $G$ is an $N R$-subgroup of $G$ (see [16]). A subgroup $H$ of a group $G$ is said to be pronormal in $G$ if for every $g \in G, H$ and $H^{g}$ are conjugate in their join $\left\langle H, H^{g}\right\rangle$. A group $G$ satisfies $\mathbf{H}_{p}$ if every normal subgroup of a Sylow $p$-subgroup of $G$ is pronormal in $G[1,7]$. The following lemmas show the relations between the $\overline{N E}_{p^{-}}, N R_{p^{-}}, \mathbf{H}_{p^{-}}, \mathcal{Y}_{p^{-}}$and $\mathcal{X}_{p}$-properties.

Lemma 2.1. Let $G$ be a group and let $p$ be a prime.

(1) If $G$ satisfies $\overline{N E}_{p}$, then $G$ satisfies $N R_{p}$.

(2) If $G$ satisfies $N R_{p}$, then $G$ satisfies $\mathbf{H}_{p}$. 
Proof. Let $P$ be a Sylow $p$-subgroup of $G$ and let $H \unlhd P$.

(1) By the assumptions, since $P \cap H^{G} \leqslant N_{G}(H) \cap H^{G}=H$ it follows that $G$ satisfies $N R_{p}$.

(2) This is Proposition 2.8(2) from [16].

Lemma 2.2. Let $G$ be a group and let $p$ be a prime. Then:

(1) $G$ satisfies $\mathcal{Y}_{p}$ if and only if every subgroup of $G$ satisfies $\mathbf{H}_{p}[5$, Corollary $2.2 .24]$

(2) $G$ satisfies $\mathcal{X}_{p}$ if and only if $G$ satisfies $\mathbf{H}_{p}$ and $G$ has Iwasawa Sylow p-subgroups [7, Theorem D].

Lemma 2.3. [16, Theorems 2.10-2.11] Let $p$ be a prime and let $G$ be a $p$ soluble group. Then:

(1) $G$ satisfies $\mathbf{H}_{p}$ if and only if $G$ satisfies $N R_{p}$;

(2) every subgroup of $G$ satisfies $\mathbf{H}_{p}$ if and only if every subgroup of $G$ satisfies $N R_{p}$.

By Lemmas 2.2 and 2.3 we obtain the following corollaries.

Corollary 2.4. Let $p$ be a prime and let $G$ be a p-soluble group. Then:

(1) $G$ satisfies $\mathcal{Y}_{p}$ if and only if every subgroup of $G$ satisfies $N R_{p}$.

(2) $G$ satisfies $\mathcal{X}_{p}$ if and only if $G$ satisfies $N R_{p}$ and $G$ has Iwasawa Sylow p-subgroups.

Examples 2.1-2.3 indicate differences between the classes of groups used in characterizations of soluble $P S T$ - and PT-groups in Theorem 1.1 and the ones in Theorems 2.6-2.7. Examples 2.1-2.2 show that the converse of Lemma 2.1(1) does not hold (even in the soluble universe).

Example 2.1. Let $G=A_{5}$, the alternating group of degree 5 . Then $G$ satisfies $\mathcal{Y}_{5}$ and every subgroup of $G$ satisfies $N R_{5}$. We will show that $G$ does not satisfy $\overline{N E}_{5}$. Let $H=\langle(12345)\rangle$. Then $\left|N_{G}(H)\right|=10$ and $H^{G} \cap N_{G}(H)=$ $N_{G}(H) \neq H$, therefore $H$ is not an $N E$-subgroup of $G$.

Example 2.2. Let $G$ be the semidirect product of a quaternion group $P$ of order 8 with a cyclic group $Q$ of order 3 , which induces the automorphism permuting cyclically the three maximal subgroups of the quaternion group. Then $G$ satisfies $\mathcal{Y}_{3}$ and every subgroup of $G$ satisfies $N R_{3}$. We will show that $G$ does not satisfy $\overline{N E}_{3}$. Since $Q^{G} \cap N_{G}(Q)=G \cap Q P^{\prime}=Q P^{\prime} \neq Q, Q$ is not an $N E$-subgroup of $G$. 
The following example shows that the converse of Lemma 2.1(2) does not hold for non- $p$-soluble groups. It also shows that Corollary 2.4 is not true for non- $p$-soluble groups.

Example 2.3. Let $G=P S L(2,53)$. Then a Sylow 3-subgroup of $G$ is cyclic of order $3^{3}$. Since a normalizer of any nontrivial 3-subgroup of $G$ is dihedral of order $2 \cdot 3^{3}, G$ satisfies $\mathcal{C}_{3}$, so it satisfies $\mathcal{Y}_{3}$. Let $H$ be a subgroup of order $3^{2}$ and $P$ be a Sylow 3-subgroup of $G$ such that $H<P$. Then $H^{G} \cap N_{G}(H)=$ $N_{G}(H) \neq H$ and $H^{G} \cap P=P \neq H$. Therefore neither $G$ satisfies $N R_{3}$ nor $G$ satisfies $\overline{N E}_{3}$.

The following example shows that the class of all $N R_{p}$-groups $\left(\overline{N E}_{p}\right.$-groups) is not closed under taking subgroups.

Example 2.4. Let $p$ be an odd prime and let $P=\langle a, b, c| a^{p}=b^{p}=c^{p}=$ $1,[a, b]=c\rangle$ be an extraspecial group of order $p^{3}$ and exponent $p$. Let $x$ be the automorphism of $P$ order 2 given by $a^{x}=a^{-1}, b^{x}=b^{-1}$. Let $G=P \rtimes\langle x\rangle$ be the corresponding semidirect product. It is easily seen that $G$ satisfies $N R_{p}$ and $\overline{N E}_{p}$. Let $H=\langle b, c, x\rangle$. Then $\langle b, c\rangle$ is a Sylow $p$-subgroup of $H$, $\langle b c\rangle \triangleleft\langle b, c\rangle$ but $(\langle b c\rangle)^{H} \cap\langle b, c\rangle=\langle b, c\rangle$, so $H$ does not satisfy $N R_{p}$. Therefore $H$ does not satisfy $\overline{N E}_{p}$.

Theorem 2.5. If $G$ is a soluble PST-group, then $G$ satisfies $\overline{N E}_{p}$ for all $p \in \pi(G)$.

Proof. Let $G$ be a soluble PST-group. Assume that $p \in \pi(G), P$ is a Sylow $p$-subgroup of $G$ and $H$ is a normal subgroup of $P$. We will show that $N_{G}(H) \cap$ $H^{G}=H$.

Let $L$ the nilpotent residual of $G$. By [5, Theorem 2.1.8] $L$ is an abelian Hall subgroup of odd order of $G$ in which $G$ acts by conjugation as a group of power automorphisms. Therefore $G=L M$, where $M$ is a nilpotent Hall subgroup of $G$ and $L \cap M=1$. Let $p \in \pi(L)$. Since every subgroup of $L$ is normal in $G$, it follows that $G$ satisfies $\overline{N E}_{p}$. Therefore we can assume that $P \leqslant M$. Then $H$ is normal in $M$. Hence $N_{G}(H)=N_{L}(H) M$ and $H^{G}=H^{L}=[L, H] H$. Since $L \cap M=1$, it follows that $N_{L}(H)=C_{L}(H)$. By [12, III.13.4] we have that $L=[L, H] \times C_{L}(H)$. Consequently, $N_{G}(H) \cap H^{G}=C_{L}(H) M \cap[L, H] H=$ $\left(C_{L}(H) M \cap[L, H]\right) H=\left(C_{L}(H) \cap[L, H]\right) H=H$. This ends the proof.

Theorem 2.6. Let $G$ be a group. The following conditions are equivalent:

(1) G is a soluble PST-group;

(2) every subgroup of $G$ satisfies $\overline{N E}_{p}$ for all $p \in \pi(G)$;

(3) every subgroup of $G$ satisfies $N R_{p}$ for all $p \in \pi(G)$. 
Proof. $(1) \Rightarrow(2)$ Since the class of all soluble $P S T$-groups is closed under taking subgroups by Corollary 2.1.9 from [5] it follows that $G$ and its subgroups satisfy $\overline{N E}_{p}$ for all $p \in \pi(G)$ by Theorem 2.5 .

$(2) \Rightarrow(3)$ Follows by Lemma $2.1(1)$.

$(3) \Rightarrow(1)$ By Lemmas 2.1(2) and 2.2(1) $G$ satisfies $\mathcal{Y}_{p}$ for every $p \in \pi(G)$.

Hence $G$ is a soluble $P S T$-group by Theorem $1.1(2)$.

Theorem 2.7. Let $G$ be a group. The following conditions are equivalent:

(1) G is a soluble PT-group;

(2) $G$ satisfies $\overline{N E}_{p}$ and $G$ has Iwasawa Sylow $p$-subgroups for every $p \in$ $\pi(G)$;

(3) $G$ satisfies $N R_{p}$ and $G$ has Iwasawa Sylow $p$-subgroups for every $p \in$ $\pi(G)$;

Proof. $(1) \Rightarrow(2)$ Follows by Theorems 2.5 and 1.1(3).

$(2) \Rightarrow(3)$ Follows by Lemma $2.1(1)$.

$(3) \Rightarrow(1)$ Follows by Lemmas $2.1(2), 2.2(2)$ and Theorem 1.1(1).

\section{Groups in which normality is transitive}

For a subgroup $K$ of $G, K s n G$ stands for " $K$ is subnormal in $G$ ". The Wielandt subgroup of a group $G$, denoted by $\omega(G)$, is the intersection of the normalizers of all subnormal subgroups of $G$. Let $\operatorname{Soc}(G)$ denote the socle of $G$, i.e. the subgroup generated by all minimal normal subgroups of $G$. In [20] Wielandt proved that $\omega(G) \geqslant \operatorname{Soc}(G)$ for every group $G$, hence in partcular $\omega(G)>1$ whenever $G>1$. Groups $G$ satisfying $\omega(G)=G$ are $T$-groups.

Let $p$ be a prime. A $p^{\prime}$-perfect group is one with no non-trivial factor groups of order coprime to $p$. In [10] Bryce and Cossey defined $\omega^{p}(G)$, the local Wielandt subgroup of a group $G$, to be the intersection of the normalizers of all $p^{\prime}$-perfect subnormal subgroups of $G$. They showed that for a group $G$, $\omega(G)=\bigcap_{p \in \pi(G)} \omega^{p}(G)$ (they proved it for soluble groups, but the same proof is true for all groups). Groups $G$ satisfying $G=\omega^{p}(G)$ are groups for which every $p^{\prime}$-perfect subnormal subgroup is normal. Such groups are called $\mathcal{T}_{p}$-groups. In the same paper Bryce and Cossey showed that in the soluble universe the classes $\mathcal{T}_{p}$ are local definitions of the local class of all $T$-groups.

In [13] Kaplan introduced the following notion.

Definition 3.1. ([13]) Let $G$ be a group. We define $\omega^{*}(G)$ to be the set of all elements $x \in G$ with the following properties:

$$
x \in N_{G}(H) \Rightarrow x \in N_{G}(K) \text { for each } K \triangleleft H \leqslant G .
$$


By definition $\omega^{*}(G)$ is a subset of $\omega(G)$. In [13] Kaplan showed that $G$ is a soluble $T$-group if and only if $\omega^{*}(G)=G$. A $p$-soluble group $G$ has $p$ length one $\left(l_{p}(G)=1\right)$ if and only if for every prime $p, G / O_{p^{\prime}}(G)$ has a normal Sylow $p$-subgroup (see [19]). Kaplan also proved that if $G$ is a metanilpotent group, then $\omega(G)=\omega^{*}(G)$. It is easy to see that a metanilpotent group has $p$-length one for all primes $p$. In [8] Beidleman and Heineken proved that every soluble $N N S$-group (group in which all subnormal subgroups have subnormal normalizers) is of $p$-length one for all primes $p$. They showed also that a soluble $N N S$-group need not be metanilpotent.

It can been seen that Kaplan's result [13, Theorem 1] related to the Wielandt subgroups of metabelian groups extends to the class of all soluble groups of $p$-length one for all $p$. We will need the following lemmas.

Lemma 3.1. ([13, Lemma 2.1]) Let $G$ be a group. Then $x \in \omega^{*}(G)$ if and only if $x \in \omega(H)$ for each subgroup $H \leqslant G$ such that $x \in H$.

Lemma 3.2. ([2, 3.4] and $[19,5.40])$ A group $G$ has p-length one for all primes $p$ if and only if $H N / N \cap \omega(G / N) \leqslant \omega(H N / N)$ for all $H \leqslant G$ and $N \unlhd G$.

Corollary 3.3. Let $G$ be a group $G$ of p-length one for all primes $p$. Then $\omega^{*}(G)=\omega(G)$.

Proof. We should only prove that $\omega(G) \subseteq \omega^{*}(G)$. Let $x \in \omega(G) \backslash \omega^{*}(G)$. Let $A$ be any subgroup of $G$ such that $x \in A$. By Lemma $3.2 A \cap \omega(G) \leqslant \omega(A)$. Hence $x \in \omega(A)$ for any $A \leqslant G$ such that $x \in A$. By Lemma 3.1 it follows that $x \in \omega^{*}(G)$, a contradiction.

There exists a soluble group $G$ with $l_{p}(G)>1$ for some $p$ such that $\omega^{*}(G) \neq$ $\omega(G)$. Namely $l_{2}\left(S_{4}\right)=2, \omega^{*}\left(S_{4}\right)=1$ and $\omega\left(S_{4}\right)=\operatorname{Soc}\left(S_{4}\right)$.

Definition 3.2. Let $G$ be a group and let $p$ be a prime. We define $\omega_{p}^{*}(G)$ to be the set of all elements $x$ of $G$ with the following property:

$x \in N_{G}(H) \Rightarrow x \in N_{G}(K)$ for each $p^{\prime}$-perfect subgroup $K$ of $G$ and $H \leqslant G$ such that $K$ sn $H$.

It is clear by definition that $\omega_{p}^{*}(G)$ is a subset of $\omega^{p}(G)$. Notice that $x \in \omega_{p}^{*}(G)$ implies $x \in \omega_{p}^{*}(A)$ for each $A \leqslant G$ containing $x$. Example 3.1 shows that $\omega_{p}^{*}(G)$ need not be a subgroup of $G$.

Lemma 3.4. Let $G$ be a group and let $p$ be a prime. Then $x \in \omega_{p}^{*}(G)$ if and only if $x \in \omega^{p}(H)$ for each subgroup $H \leqslant G$ such that $x \in H$. 
Proof. Assume first that $x \in \omega_{p}^{*}(G)$ and let $x \in H \leqslant G$. Suppose that $K$ is a subnormal $p^{\prime}$-perfect subgroup of $H$. Since $x \in H \leqslant N_{G}(H)$ and $x \in \omega_{p}^{*}(G)$ we have $x \in N_{G}(K)$. Therefore $x \in \omega^{p}(H)$.

For the other direction assume that $x \in \omega^{p}(H)$ for each $H \leqslant G$ such that $x \in H$. Suppose that $K$ sn $L \leqslant G, K$ is a $p^{\prime}$-perfect subgroup of $G$ and $x \in N_{G}(L)$. Then $x \in \omega^{p}\left(N_{G}(L)\right)$ and since $K s n N_{G}(L)$ we have that $x \in N_{G}(K)$. Therefore $x \in \omega_{p}^{*}(G)$.

Lemma 3.5. Let $G$ be a group. Then $\omega^{*}(G)=\bigcap_{p \in \pi(G)} \omega_{p}^{*}(G)$.

Proof. It is an immediate consequence of the definitions that $\omega^{*}(G) \leqslant \omega_{p}^{*}(G)$ for every prime $p$.

For the other direction, set $W=\bigcap_{p \in \pi(G)} \omega_{p}^{*}(G)$ and let $K \triangleleft H \leqslant G$. Now $K$ can be written as a product of subnormal subgroups of $H$ each of which has a unique maximal normal subgroup and hence is $p^{\prime}$-perfect for some $p$. But all such subgroups are normalized by $W$ and so $W \leqslant \omega^{*}(G)$.

Example 3.1. Kaplan showed that for a group $G, \omega^{*}(G)$ does not have to be a subgroup of $G$. Namely, for the alternating group $A_{5}$, the set $\omega^{*}\left(A_{5}\right)$ is the set of all elements of $G$ with order distinct from 3 . We have $\omega^{*}\left(A_{5}\right) \leqslant \omega_{2}^{*}\left(A_{5}\right)$. We set $H=\langle(1,2)(3,4),(1,3)(2,4)\rangle$ and $K=\langle(1,2)(3,4)\rangle$. Then $(1,2,3) \in$ $N_{A_{4}}(H) \backslash N_{A_{4}}(K)$. Similar considerations give $\omega^{*}\left(A_{5}\right)=\omega_{2}^{*}\left(A_{5}\right)$.

In the following Theorem we use some observations from [3].

Theorem 3.6. Let $G$ be a group and let $p$ be a prime. Then $G$ satisfies $\mathcal{C}_{p}$ if and only if $G=\omega_{p}^{*}(G)$.

Proof. Clearly, if $G=\omega_{p}^{*}(G)$, then $G$ satisfies $\mathcal{C}_{p}$.

Conversely, assume that $K$ sn $H \leqslant G$ and $K$ is a $p^{\prime}$-perfect subgroup of $G$. We will show that $K \unlhd N_{G}(H)$. Since the property $\mathcal{C}_{p}$ is subgroup-closed it is enough to consider the case $K \unlhd H \unlhd G$. Let $K_{p}$ be a Sylow $p$-subgroup of $K$ and $H_{p}$ be a Sylow $p$-subgroup of $H$ such that $K_{p} \leqslant H_{p}$. Since $G$ satisfies $\mathcal{C}_{p}$, we have that $K_{p} \unlhd N_{G}\left(H_{p}\right)$. Hence by the Frattini argument we obtain $G=H N_{G}\left(H_{p}\right)=K N_{H}\left(K_{p}\right) N_{G}\left(H_{p}\right)=K N_{G}\left(K_{p}\right)$. Hence, since $K$ is $p^{\prime}$-perfect it follows that $K_{p}^{G}=K_{p}^{K}=K$. Therefore $K$ is normal in $G$. This ends the proof.

Corollary 3.7. Let $G$ be a group. Then $G$ is a soluble T-group if and only if $G=\omega_{p}^{*}(G)$ for every $p \in \pi(G)$.

Proof. Follows by Theorem 3.6 and [5, Theorem 2.2.2]. 


\section{Conclusions and further research}

All our previous results show that investigation of classes of groups by using subgroup embedding properties is still fruitful. We have used $N E$-subgroups, $N R$-subgroups and the generalization of the local Wielandt subgroup to characterize soluble $T$-, $P T$ - and $P S T$-groups. In the literature these subgroups were also applied to obtain characterizations and criteria for a group to belong to a particular class of groups (see for example $[9,14]$ ). They will surely be the subject to some further research.

Finally, we mention five open problems concerning to this topic.

Problem 1. Find all groups $G$ such that $\omega^{*}(G)=\omega(G)$.

Problem 2. Let $G$ be a group. Study the structures of $G$ and $\omega^{*}(G)$ when $\omega^{*}(G)$ is a subgroup of $G$.

Problem 3. Let $p$ be a prime. Find all groups $G$ such that $\omega_{p}^{*}(G)=\omega^{p}(G)$.

Problem 4. Let $p$ be a prime and let $G$ be a group. Study the structures of $G$ and $\omega_{p}^{*}(G)$ when $\omega_{p}^{*}(G)$ is a subgroup of $G$.

We say that a group $G$ satisfies $N E_{p}$ if every maximal subgroup of a Sylow $p$-subgroup of $G$ is an $N E$-subgroup of $G$. It can be proved that $G$ is a soluble $P S T$-group if and only if every subgroup of $G$ satisfies $N E_{p}$ for all $p \in \pi(G)$.

Problem 5. Is it true that $G$ is a soluble $P T$-group if and only if $G$ satisfies $N E_{p}$ and has Iwasawa Sylow $p$-subgroups for every $p \in \pi(G)$ ?

\section{References}

[1] Alejandre M.J., Ballester-Bolinches A., Pedraza-Aguilera M.C., Finite soluble groups with permutable subnormal subgroups, J. Algebra 240 (2001), 705-722.

[2] Ali A., Groups of p-length one for all primes p, Comm. Alg. 26 (9) (1998), 2895-2904.

[3] Ballester-Bolinches A., Esteban-Romero R., On finite T-groups, J. Aust. Math. Soc. 75 (2003), 181-191.

[4] Ballester-Bolinches A., Esteban-Romero R., Sylow permutable subnormal subgroups of finite groups, J. Algebra 251 (2002), 727-738 .

[5] Ballester-Bolinches A., Esteban-Romero R., Asaad M., Products of finite groups, Walter de Gruyter GmbH\& Co, KG, Berlin/New York, 2010. 
[6] Bauman S., The intersection map of subgroups, Arch. Math. 25 (1974), $337-340$.

[7] Beidleman J.C., Brewster B., Robinson D.J.S., Criteria for permutability to be transitive in finite groups, J. Algebra 222 (1999), 400-412.

[8] Beidleman J.C., Heineken H., Groups with subnormal normalizers of subnormal subgroups, Bull. Aust. Math. 86 (2012), 11-21.

[9] Berkovich Y., Subgroups with the character restriction property and related topics, Houston J. Math. 24 (1998), 631-638.

[10] Bryce R.A., Cossey J., The Wielandt subgroup of a finite soluble group, J. London Math. Soc. (2)40 (1989), 244-256.

[11] Grätzer G., Universal algebra, Springer, New York, 2008.

[12] Huppert B., Endliche Gruppen I, Springer-Verlag, Berlin-New York, 1967.

[13] Kaplan G., On finite T-groups and the Wielandt subgroup, J. Group Theory $\mathbf{1 4}(2011)$, 855-863.

[14] Li S., On minimal non-PE-groups, J. Pure Appl. Algebra 132 (1998), $149-158$.

[15] Li Y., Finite groups with NE-subgroups, J. Group Theory 9 (2006), 49-58.

[16] Malinowska I.A., Finite groups with NR-subgroups or their generalizations, J. Group Theory 15, no. 5 (2012), 687-707.

[17] Malinowska I.A. Finite groups with some $N R$-subgroups or $\mathcal{H}$-subgroups, Monatsh. Math. 171 (2013), 205-216.

[18] Robinson D.J.S., A Course in the Theory of Groups, Springer-Verlag, New York, 1996.

[19] Wetherell C.J.T., Subnormal structure of finite soluble groups, (Ph.D. thesis) Australian National University, ACT, Australia, http:// thesis.anu.edu.au. (2001).

[20] Wielandt H., Über den Normalizer der Subnormale Untergruppen, Math. Z. 69 (1958), 463-465.

Izabela Agata MALINOWSKA,

Institute of Mathematics,

University of Białystok,

15-267 Białystok, Akademicka 2, Poland.

Email: izabelam@math.uwb.edu.pl 